\title{
Human Motion Detection and Tracking for Real-Time Security System
}

\author{
Pavithra $\mathbf{S}^{1}$, Mahanthesh $\mathbf{U}^{1}$, Stafford Michahial ${ }^{1}$, Dr. M Shivakumar ${ }^{2}$ \\ Asst. Professor, Department of IT $^{1}$ \\ Prof \& Head, Department of IT ${ }^{2}$
}

\begin{abstract}
People detection and tracking is one of the important research fields that have gained a lot of attention in the last few years. Although person detection and counting systems are commercially available today, there is a need for further research to address the challenges of real world scenarios. There is lot of surveillance cameras installed around us but there are no means to monitor all of them continuously. It is necessary to develop a computer vision based technologies that automatically process those images in order to detect problematic situations or unusual behavior. Automated video surveillance system addresses real-time observation of people within a busy environment leading to the description of their actions and interactions. It requires detection and tracking of people to ensure security, safety and site management. Object detection is one of the fundamental steps in automated video surveillance. Object detection from the video sequence is mainly performed by background subtraction technique. It is widely used approach for detecting moving objects from static cameras. As the name suggests, background subtraction is the process of separating out the foreground objects from the background in a sequence of video frames. The main aim of the surveillance system here is, to detect and track an object in motion by using single camera. Camera is fixed at the required place background subtraction algorithm is used for segmenting moving object in video. If human entity is detected the tracking lines are formed around human and the object is tracked. The system when realizes the human entry, it is processed in a second and the alert is produced for the security purpose. The main aim is to develop a realtime security system.
\end{abstract}

Keywords: Video surveillance system, Moving object detection, Tracking, Background subtraction, Producing alarm.

\section{INTRODUCTION}

Surveillance is the monitoring of the behavior, activities, or other changing information, usually of people for the purpose of influencing, managing, directing, or protecting them. This can include observation from a distance by means of electronic equipment or interception of electronically transmitted information and it can refer to simple, relatively no-or low-technology methods such as human intelligence agents and postal interception. The word surveillance comes from a French phrase for "watching over". Surveillance is very useful to governments and law enforcement to maintain social control, recognize and monitor threats, and prevent/investigate criminal activity.

With the advent of programs such as the Total Information Awareness program and ADVISE, technologies such as high speed surveillance computers and biometrics software, and laws such as the Communications Assistance for Law Enforcement Act, governments now possess an unprecedented ability to monitor the activities of their subjects [16]. Surveillance cameras are video cameras used for the purpose of observing an area. They are often connected to a recording device or IP network, and may be watched by a security guard or law enforcement officer and also in banks and where security is concerned. Cameras and recording equipment used to be relatively expensive and required human personnel to monitor camera footage, but analysis of footage has been made easier by automated software that organizes digital video footage into a searchable database, and by video analysis software (such as VIRAT and Human ID). The amount of footage is also drastically reduced by motion sensors which only record when motion is detected. With cheaper production techniques, surveillance cameras are simple and inexpensive enough to be used in home security systems, and for everyday surveillance. The first generation of video surveillance systems (1980's) is the traditional analog closed-circuit television (CCTV) network. In the system, analog video cameras are connected by coaxial cables to surveillance screens for monitoring by human operators or the cameras could be connected to videotape recorders for archiving purposes.

The second generation video surveillance (1990's) replaced the videotape recorder with a digital video recorder (DVR) with the data archived on hard drives. More recent systems have network connections so the video data can be stored on servers. The third generation is an IP network system, where the data is continuously being transmitted over the network. There are some excellent survey papers in the literature on video surveillance systems. The first CCTV system was developed as far back as the 1940's when it was used by the military in Germany to observe the launch of V2 
Vol. 5, Issue 12, December 2016

rockets. CCTV systems were also used by the US military to develop and test atomic weapons, as this allowed one to observe the tests from a safe distance. By the 1970's, CCTV had become common in the commercial world. Video surveillance was used in stores and banks to protect their properties and assets. Today, surveillance cameras are omnipresent, from security sensitive places, such as borders and military bases for migrating terrorist activities, to private homes for burglary prevention. The large quantity of data involved makes it infeasible to assure vigilant monitoring by manual operators for long period of time due to monotony and fatigue. As a result, video feeds are usually achieved for forensic purposes in the event suspicious activities take place. In order to assist manual operators with identification of important tasks in videos, an intelligent surveillance system can be used. We proposed a method for moving object detection and tracking with visual surveillance application and consider computational cost as the major design goal of our work. Moving object detection aims at extracting moving objects that are of interest in video sequences. The problems with dynamic environmental conditions make moving object detection very challenging. Commonly used techniques for moving object detection are background subtraction, temporal frame differencing, and optical flow. The next step in the video analysis is object tracking. This problem can be formulated as a hidden state estimation problem given available observations. Another way to look at object tracking is the creation of temporal correspondence among detected object from frame to frame. Object tracking is an important component of many vision systems. It is send not only for visual surveillance, but also for augmented reality, traffic control, medical imaging, gesture recognition, and video. The final step of an "intelligent" visual surveillance system is to analyze the content of the video and to identify important events in a scene. This step provides human operators with high level analyses to assist them with making their decisions more accurately, effectively, and efficiently. In this paper, we present an automated video surveillance system for realtime security system. Here we use a single camera for detecting the human motion and tracking it over time. The main goal is to detect the object efficiently using background subtraction techniques, aims to reduce the cost and to increase efficiency in the security systems.

This paper is divided into the following sections. In section II we discuss about previous studies related to our subject and discussion; in section III we describeabout the proposed work and finally section IV shows the result analysis.

\section{REVIEW ABOUT PREVIOUS WORKS}

Numerous studies have been done based on people counting using computer vision, [1] in this paper they present a solution to bidirectional people counting based on information provided by an overhead stereo system, four fundamental aspects are identified:
1. The detection and tracking of human motion using an extended particle filter

2. Use of 3-D measurements in order to increase the system's robustness

3. Modified K-means algorithm is used to provide a deterministic output

4. People trajectories are generated in order to perform people counting.

As a result, the stereo counting system produces a certain percentage of error. They are due to the following reasons: detection problem, slow movement problem, deterioration problem. [2] it aims to develop an effective method for calculating the number of people and locate each individual in a low resolution image with complicated scenes. Some people counting system is based on face detection technique [3] since the camera is placed at the head height they present the people counting approach based on face detection; face trajectory an angle histogram of neighbouring points is extracted. Finally, an Earth Mover's Distance-based K-NN classification discriminates true face trajectories from the false ones. In automated video analysis object detection is based on background subtraction or DECOLORS technique which is shown in paper [4]. DECOLOR out performs the state-of-the-art approach and it can work effectively on a wide range of complex scenarios.

In some system [5] the manmade objects are captured in the radar which undergoes 3-D reconstruction. Two techniques proposed: (1) MMV CS (multiple measurement vector compressive sensing), (2) Regularisation theory. [7] Frame work based on the contour based method for the object detection. It deals effectively with contour tracking for videos with abrupt motions. It out performs the particle filter-based algorithm. [8] Automatic vehicle detection system for the aerial surveillance, region based or sliding window based.

\section{PROPOSED METHODOLOGY}

In this paper we present a novel and simple method for moving object detection. The human object is captured; a data base about the pixel values are trained to the system. Video Camera is fixed at a required place where security is needed. Whenever human movement is captured by the camera it is immediately detected and the object is tracked by background subtraction method, finally the system is processed to make the alarm to produce sound (alarm). Our proposed method detects moving objects in required environments under changing illumination conditions and in the presence of background dynamics. Also present a fast implementation of the method using an extension of integral images.

The amount of footage is also drastically reduced by motion sensors which only record when motion is detected. With cheaper production techniques, surveillance cameras are simple and inexpensive enough to be used in home security systems, and for everyday surveillance. The first generation of video surveillance systems (1980's) is 
Vol. 5, Issue 12, December 2016

the traditional analog closed-circuit television (CCTV) network. The final step of an "intelligent" visual surveillance system is to analyze the content of the video and to identify important events in a scene.

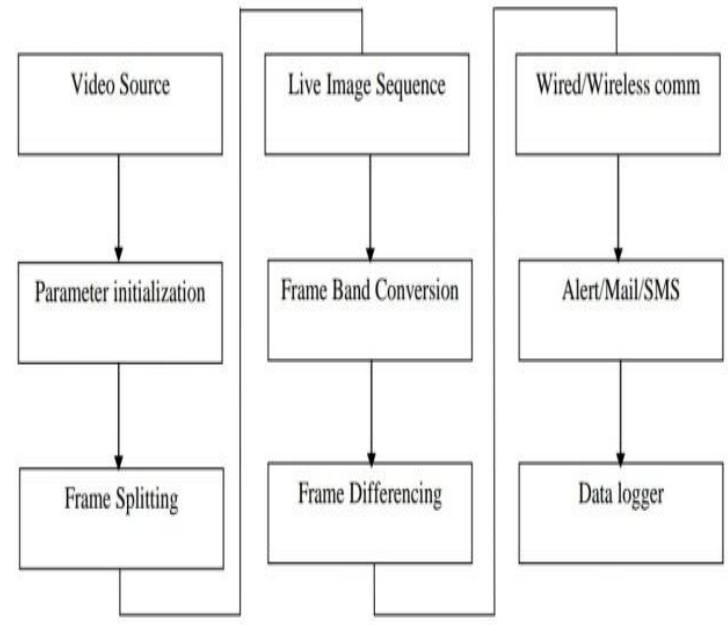

Fig.1 Block diagram of proposed work

Fig. 1 shows the overall flow for the proposed work. The object is detected using MATLAB programming using the information from the camera or web cam. Whenever the human object is detected the information is processed and this makes the system to awake and produces alert sound.A. Moving Object Detection And TrackingIn the area of moving object detection and tracking, a technique robust to background dynamics using background subtraction with adaptive pixel-wise background model update is described. A foreground-background pixel classification method using adaptive thresholding is presented. Another technique that is robust to sudden illumination changes using an illumination model and a statistical test is presented it is shown below in fig. 3 .

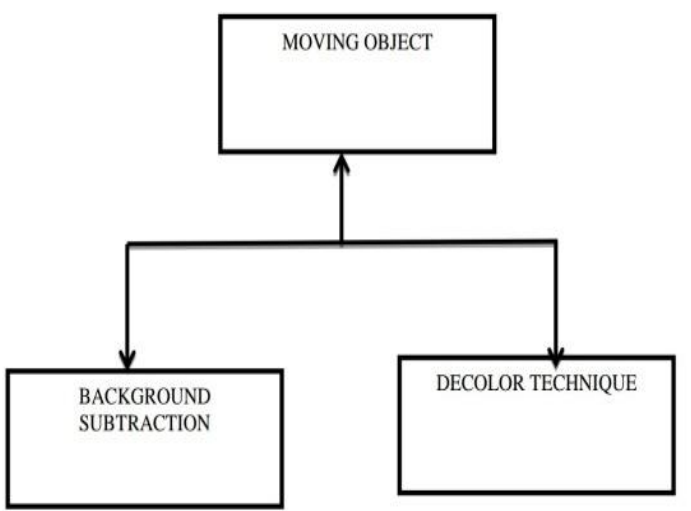

Fig.2 Moving object detection

In addition, we propose a fast method to construct an appearance model for object tracking using a particle filtering framework shown in fig. 4. Segmenting out mobile objects present in frames of a recorded video sequence is a fundamental step for many video based surveillance applications but here live video is analysed.

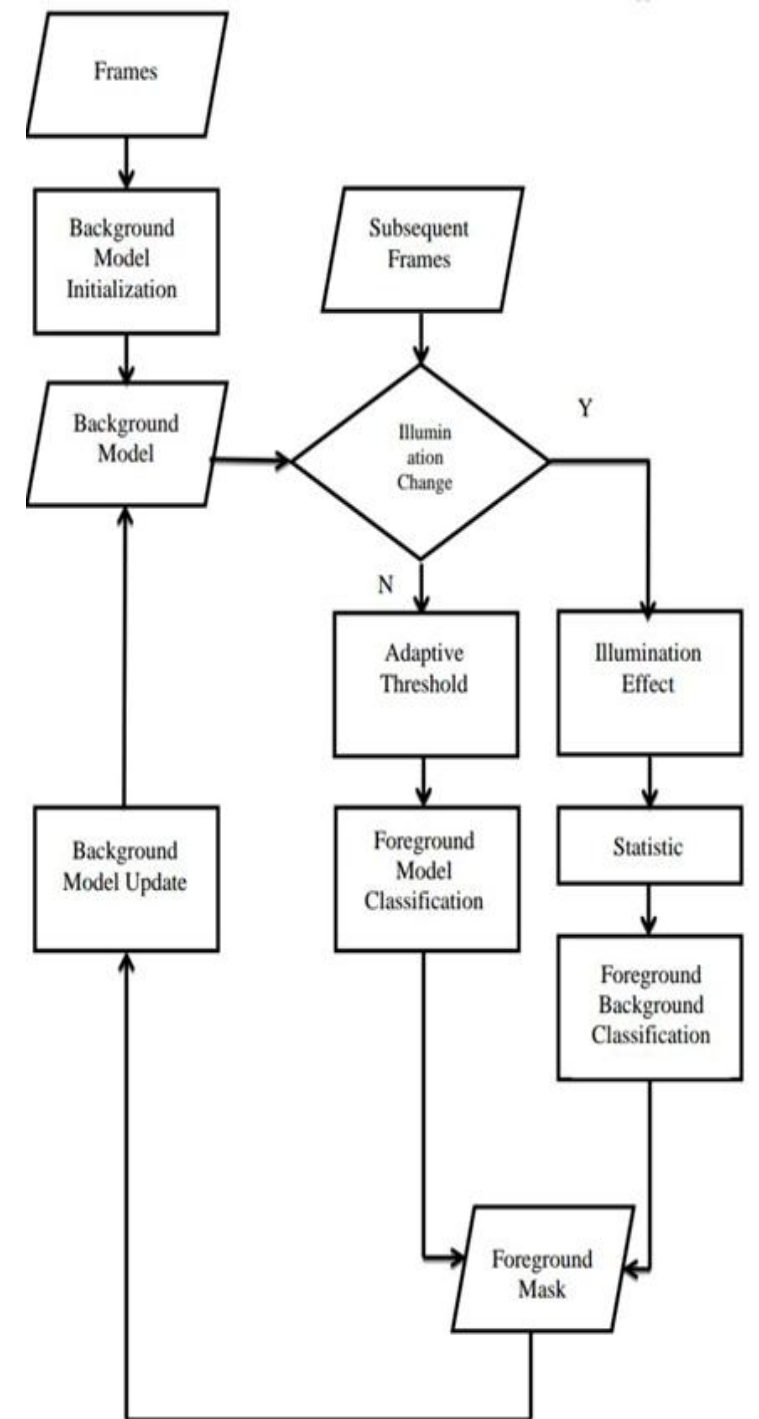

Fig.3 An overview of our proposed method for moving object detection

Here proposed a method for robust tracking with dynamic parameter setting for the likelihood model of particle filtering. As in fig.2 DECOLOR and background subtraction simultaneously performed. DECOLOR performs object detection and background estimation simultaneously with a training

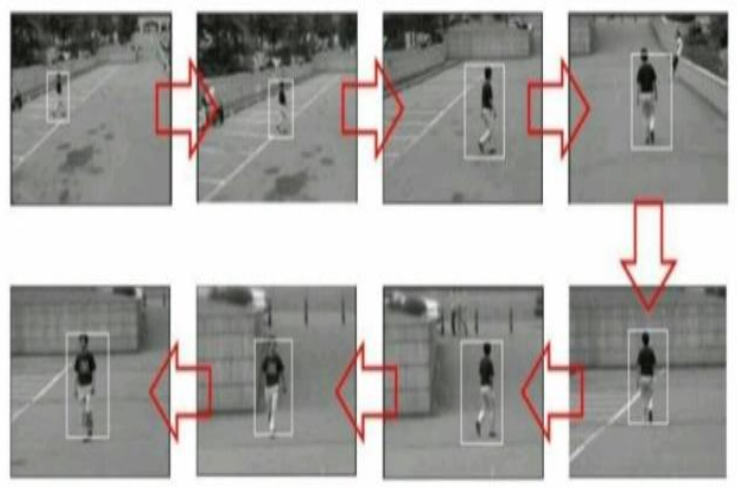

Fig.4 Tracking of moving object 
B. Background Subtraction Technique We present a novel account)and foreground/background pixel classification. and simple method for moving object detection. The The process is shown in fig. 5.

method is based on background subtraction technique. The first step, referred to as background model initialization, is to construct a background model using the initial frames. The step is based on temporal frame differencing because the background model is not available at the start of a sequence.A typical example is when a sequence starts with a moving foreground object, part of the background model will be covered and hence the background model will not be available. Once the initial background model is constructed, with each subsequent frame, we detect if there is any sudden illumination change. If there is no illumination change, simple background subtraction can be used to find the foreground pixels. The threshold used for this process is determined adaptively according to the current frame. After thresholding the difference of the pixel intensities between the background model and the current frame, a foreground object mask is generated. In the presence of sudden illumination change, we use the illumination effect, which is expressed as a ratio of pixel intensities.We determine if the pixel is a foreground or background pixel by the statistics of the illumination effect of its neighbouring pixels. A foreground object mask is generated according to this statistic. The process of obtaining the foreground mask is referred to as foreground-background pixel classification. After obtaining the foreground object mask, the background model should be updated because the scene dynamics are always evolving. We propose a technique that updates the background model according to two factors: how long a pixel has been a background pixel, and how large the value of the pixel is in the difference frame.

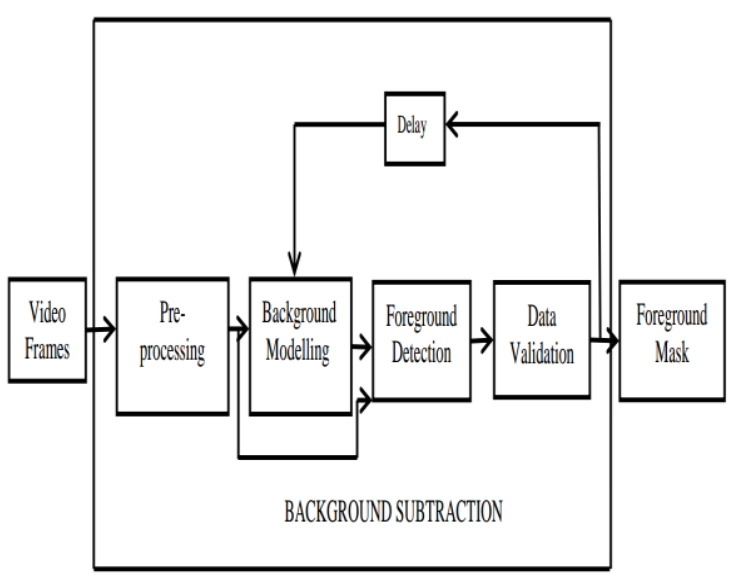

Fig.5 Background Subtraction

Background subtraction is a widely used for detecting moving objects. The ultimate goal is to "subtract" the background pixels in a scene leaving only the foreground objects of interest. If one has a model of how the background pixels behave the "subtraction" process is very simple Background subtraction usually consists of three attributes besides the basic structure of the background model, background initialization background maintenance (updating the background model to

\section{RESULTS AND DISCUSSION}

A. Background Subtraction Algorithm As the name suggests, background subtraction is the process of separating out foreground objects from the background in a sequence of video frames. Background subtraction is widely used approach for detecting moving objects from static cameras.The four major steps in a background subtraction algorithm are, (it is shown in fig. 5 above)

1.Pre-processing,

2.Background modelling,

3.Foreground detection and

4.Data validation.

Background modelling is the heart of any background subtraction algorithm. Background model is robust against environmental changes in the background, but sensitive enough to identify all moving objects of interest. Background modelling is divided into two types: (1) Nonrecursive and (2) Recursive. A non-recursive technique uses a sliding window approach for background estimation. Non-recursive is highly adaptive as they do not depend on the history beyond those frames stored in the buffer. Some of the commonly used non-recursive techniques are: frame differencing, median filter, mean filter. Here in our proposed work we use frame differencing method for background modelling.B. Frame Differencing Fundamental logic for detecting moving objects from the difference between the current frame and a reference frame is called background image subtraction and this method is called as frame differencing

Frame difference: | framei - framei-1 $\mid>$ Th

Where: Th - Threshold.

The estimated background is just the previous frame. It evidently works only in particular conditions which is very sensitive to the threshold (Th).Background is estimated to be the previous frame. Background subtraction equation then becomes:

$$
\mathrm{B}(\mathrm{x}, \mathrm{y}, \mathrm{t})=\mathrm{I}(\mathrm{x}, \mathrm{y}, \mathrm{t}-1) \square|\mathrm{I}(\mathrm{x}, \mathrm{y}, \mathrm{t})-\mathrm{I}(\mathrm{x}, \mathrm{y}, \mathrm{t}-1)|>\mathrm{Th}
$$

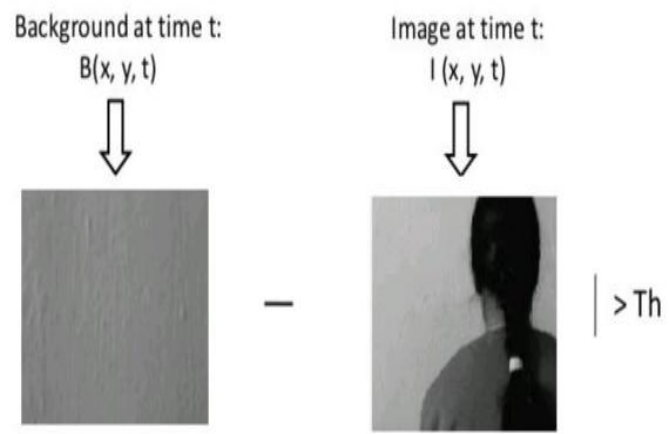

Fig.6 Frame differencing

Frame differencing involves the following steps:

1.Estimate the background for time $t$.

2.Subtract the estimated background from the input frame. 
3.Apply the threshold (Th), to the absolute difference to get the foreground mask.

The key parameter in the thresholding process is the choice of the threshold value. Several different methods for choosing a threshold value are: (1) the users can manually choose a threshold value, or (2) a thresholding algorithm can compute a value automatically which is known as automatic thresholding.C. Simulation Result The object detection is done efficiently using background subtraction technique. We use MATLAB 7.14(R2012A) software for this purpose. The fig. 7 shows the output window with the static background, here no human entry is found and in the background subtraction map no difference is found.
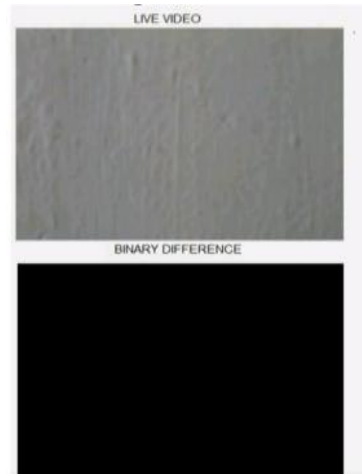

Fig.7 Output window when no human is detected

The fig 8 shows detection of human and when human entry is found the tracking lines are formed over the image by background subtraction technique.
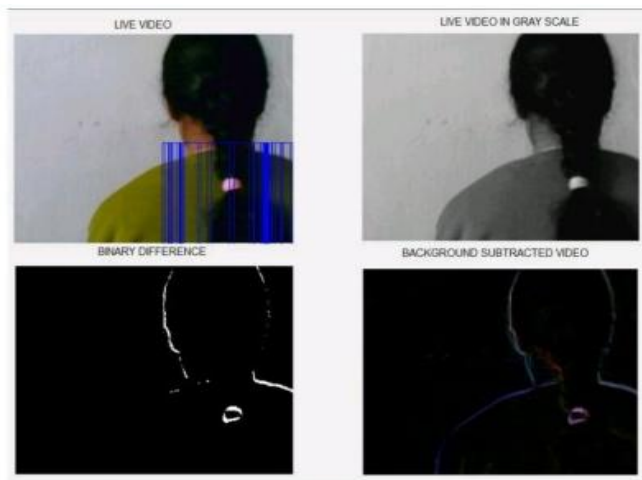

Fig.8 Output window when human is detected and formation of tracking lines

\section{CONCLUSION}

In this paper, human motion detection and tracking for real time security system was formulated. Video surveillance is focused on people counting and tracking mainly for realtime applications such as security system, traffic monitoring, etc. The object is detected from the live video and tracked using background subtraction, this system is proposed for real-time security purpose. In the live video 18 frames are processed at a unit time. Based on the camera's range the monitoring area may be increased. It is mainly applicable in banks, jewellery shops, military etc. Object detection is made efficiently using the background subtraction technique and the frames processed per second are improved. For this purpose MATLAB 7.14(R2012A) tool is used. This system increases the efficiency and reduces the cost and total time consumed. Thus the realtime security system is built efficiently.

\section{REFERENCES}

[1] Jorge Garcia, Alfredo Garden, Ignacio Bravo, Jose Luis Lazar and Miguel Martinez, (2013).“Tracking People Motion Based Extended Condensation .Algorithm, "IEEE Trans.Syst.,Man,Cybern. A, Syst., vol. 43, No. 3, May.

[2] Y.-L. Hou and G. K. H. Pang, (2011)."People counting and human detection in a challenging situation," IEEE Trans. Syst., Man, Cybern. A, Syst., Humans,vol. 41, no. 1, pp. 24-33, Jan.

[3] X. Zhao, E. Delleandrea, and L. Chen, (2009)."A people counting system based on face detection and tracking in a video," in Proc. IEEE Int. Conf. AVSS, pp. 67-72.

[4] Xiaowei Zhou, Can Yang, and Weichuan Yu, (2013). "Moving Object Detection by Detecting Contiguous Outliers in the LowRank Representation" IEEE Trans.

[5] Shi-qi Xing, Yong-zhen Li, Da-hai Dai, XueSongWang,(2012). "Three-Dimensional Reconstruction of ManMade Objects Using Polarimetric Tomographic SAR”, IEEE Trans. Vol. 51, Issue: 6 Journal on Geoscience and Remote Sensing.

[6] Liyuan Li, Weimin HuangIreneYu-Hua Gu, and. QiTian, (2004). "Statistical Modelling of Complex Backgroundsfor Foreground Object Detection" IEEE Transon Image Processing, Vol. 13, NO. 11

[7] Weiming Hu, Xue Zhou, Wei Li, WenhanLuo, Xiaoqin Zhang, and Stephen Maybank,(2013)."Active Contour-Based VisualTracking by Integrating Colors, Shapes , and Motions Image Processing", IEEETrans.onImageProcessing,Vol.22

[8] .Hsu-Yung Cheng, Chih-Chia Weng, Yi-Ying Chen, (2012). "Vehicle Detection in Aerial Surveillance Using Dynamic Bayesian Networks", IEEE Trans on Image Processing, Vol.21, Issue:4.

[9] Pushe Zhao, Hongbo Zhu, He Li, and Tadashi Shibata, (2013). "A Directional-Edge-Based Real-Time Object Tracking System Employing Multiple Candidate-Location Generation" IEEE Trans.vol. 23,no. 3, Mar.

[10] Wang. H and Suter.D, (2006). "A Novel Robust Statistical Method for Background Initialization and Visual Surveillance" Proc. Asian Conf. Computer Vision.

[11] Xinyi Cui1, Junzhou Huang2, Shaoting Zhang1, and DimitrisN,(2006)."Background Subtraction Using Low Rank and Group Sparsity Constraints" Metaxa1 CS Dept., Rutgers University Piscataway, NJ 08854, USA2 CSE Dept., Univ. of Texas at Arlington, TX, 76019, USA

[12] Michael.BHolte, Cuong Tran, Mohan. M Trivedi, Fellow, and Thomas B. Moeslund, Member, (2012). "Human Pose Estimation and Activity Recognition From Multi-View Videos: Comparative Explorations Recent Developments" VOL.6, NO. 5.

[13] Nair.V and Clark.J,(2004)."An Unsupervised, OnlineLearning Framework for Moving Object Detection,"Proc.IEEE Conf. Computer Vision and Pattern Recognition, vol. 2, pp. 317-324.

[14] Abhijitmahalanobis, Robert muise and Lockheed Martin, (2007). "Object Specific Image Reconstruction using a Compressive Sensing Architecture for Application in Surveillance System" IEEE TRANSACTION

[15] brox. T, Bruhn.V and Weickert.J, (2006)."Variational Motion Segmentation with Level Sets,"Proc. European Conf. Computer Vision. Surveillance refer from: http://news.linktv.org/topics/ surveillance

[16] S. Velipasalar, Y.-L.Tian, and A. Hampapur, (2006).“Automatic counting of interacting people by using a singleun calibrated camera," in Proc. IEEE Int Multimedia.

[17] Yang, H. H. Gonzalez-Banos, and L. J. Guibas,(2003), "Counting people in crowds with a real-time network of simple image sensors," in Proc. 9th IEEEInt.ComputVis.Conf.,pp. 122 -129. 